\title{
Applying Wearable Solutions in Dependent Environments
}

\author{
Juan A. Fraile, Javier Bajo, Juan M. Corchado, and Ajith Abraham, Senior Member, IEEE
}

\begin{abstract}
This paper proposes a multiagent system (MAS) that uses smart wearable devices and mobile technology for the care of patients in a geriatric home care facility. The system is based on an advanced ZigBee wireless sensor network (WSN) and includes location and identification microchips installed in patient clothing and caregiver uniforms. The use of radio-frequency identification and near-field communication technologies allows remote monitoring of patients, and makes it possible for them to receive treatment according to preventive medical protocol. The proposed MAS manage the infrastructure of services within the environment both efficiently and securely by reasoning, task-planning, and synchronizing the data obtained from the sensors. Additionally, this paper presents the design and implementation of the reasoning agent in the MAS. A system prototype was installed in a real environment and the results obtained are presented in this paper.
\end{abstract}

Index Terms-Dependent environment, human health, smart wearable devices, wearable systems.

\section{INTRODUCTION}

I $\mathrm{T}$ is a well-known fact that the world population is aging and that both the number of people suffering from chronic illnesses and the elderly population, in general, are rapidly increasing. New technologies, wearable devices, and communication networks are helping residents and their caregivers by providing continuous medical supervision, domotic control, rapid access to medical data, and emergency communication both in the home and in geriatric care facilities [1]-[4]. During the past several years, there has been an increase in the demand for noninvasive intelligent devices that can detect vital signs without interfering in the daily routine of those who use them [5], [6]. This demand was fueled by the need that public and private institutions have for monitoring and providing services to the dependent patients under their care.

Smart wearable devices are sensors that are designed to help and provide services to dependent persons [7]. They offer new

Manuscript received February 13, 2010; revised May 18, 2010; accepted June 14, 2010. Date of publication ; date of current version

This work was supported by the Spanish Ministry of Science and Technology under project TIN2006-14630-C03-03.

J. A. Fraile is with the University of Salamanca, 37008 Salamanca, Spain (e-mail: jafraileni@upsa.es).

J. Bajo is with the Escuela Universitaria de Informática, Pontifical University of Salamanca, 37008 Salamanca, Spain (e-mail: jbajope@ upsa.es).

J. M. Corchado is with the Faculty of Sciences and Biomedicine, Intelligent Syatems and Educational Technonlogy Research Group, University of Salamanca, 37008 Salamanca, Spain (e-mail: corchado@usal.es).

A. Abraham is with the Machine Intelligence Research Labs (MIR Labs), Scientific Network for Innovation and Research Excellence (SNIRE), Washington State, 98071, USA (e-mail: ajith.abraham@ieee.org).

Color versions of one or more of the figures in this paper are available online at http://ieeexplore.ieee.org.

Digital Object Identifier 10.1109/TITB.2010.2053849 possibilities and create new challenges as a result of the information that they supply quickly, reliably, and securely. As wireless technologies and ubiquitous computing [1] continue to unfold, network sensors [8], communication data networks [4], [9], mobile devices and smart wearable devices [6], [10], [11] make it possible to create intelligent environments and assist with longdistance medical care [3], [12], [13].

Agents and multiagent systems (MASs) have gained relevance in the development of dynamic and distributed systems, and have been successfully applied in areas, such as electronic commerce, medicine, homecare, robotics, etc. [14]-[17]. The characteristics of the agents make them suitable for developing wireless sensor network (WSN) based distributed systems. The advantage of the agents is their ability to easily adapt to the users and the characteristics of the surrounding environment [18]. Additionally, they are frequently used with mobile technology, such as general packet radio service (GPRS), nearfield communication (NFC), or Bluetooth, making it possible for them to obtain context-aware information in novel ways.

This paper describes the smart wearable device (MaRV) MAS, which is intended to automatically manage and improve the assistance to patients in geriatric facilities by using smart wearable technology. This system uses smart wearable devices to obtaincontext aware information, thus making it possible to identify, locate, and gather data for each patient. The agents are in-charge of managing and controlling the information obtained by the system. The core agent in MaRV is the head agent, which is integrated into the system. The purpose of this agent is to improve the quality of life for the user by providing efficient and relevant solutions in real time. The most important characteristics of the system are: 1) the head agent has reasoning capability, it can analyze and reason about the context data gathered by the system and provide proactive solutions; 2) the head agent can easily adapt to the context within which it acts; and (iii) gather sensor data and messages from other agents in order to provide efficient solutions. The communication between the agents and the smart wearable devices is conducted with wireless technologies: Wi-Fi, ZigBee, NFC [19], while radio-frequency identification (RFID) is used for identification [20]. These technologies provide the structure that is required for supporting the communication needs for the system agents with devices and data-handling equipment. Additionally, they increase the flexibility and efficiency of the agents, permitting them to remotely access resources (programs, teams, services, etc.) regardless of the patient's physical location.

The remainder of the paper is structured as follows: the next section presents the state-of-the-art in smart wearable devices and the way in which they interact within medical care environments. Section III describes the proposed MAS, focusing on the 
TABLE I

SENSORS USED TO DETECT VitAL SignS

\begin{tabular}{|c|c|}
\hline Sensors & Observation \\
\hline $\mathrm{ECG}$ & $\begin{array}{l}\text { Heart rate, heart rate variability } \\
(\mathrm{HRV})\end{array}$ \\
\hline EMG & Muscle activities and fatigue \\
\hline $\begin{array}{l}\text { Galvanic Skin } \\
\text { Response }\end{array}$ & Skin con ductance \\
\hline Temperature & $\begin{array}{l}\text { Skin temperature, health state (fe- } \\
\text { ver) }\end{array}$ \\
\hline Respiration & Breathing rate, physical activity \\
\hline Blood oxygen & $\begin{array}{l}\text { Status of the cardiovascular system, } \\
\text { heart rate }\end{array}$ \\
\hline Blood pressure & $\begin{array}{l}\text { Status of the cardiovascular system, } \\
\text { hypertension }\end{array}$ \\
\hline
\end{tabular}

description of how the agents interact with the smart wearable devices. Section IV presents a prototype that was developed for the care of patients in a geriatric facility. Finally, Section $\mathrm{V}$ presents the results and conclusions obtained after installing the prototype in the care facility, and suggest future areas of investigation for improving the system.

\section{Smart Wearable Devices in Health CARE ENVIRONMENTS}

Recent developments in micro and nanotechnology, the use of lightweight devices, wireless networks, and information processing have led to the emergence of noninvasive wearable devices that improve health monitoring systems [21], [22]. Wearable devices detect the state of the user within the immediate surroundings. To date, most efforts have concentrated on the detection of the physical state of the user (for example, activity, location, and physiological parameters) [6]. The data that can be provided by wearable devices and the physical state of the patients are closely related, which facilitates the process of classifying information. As a result, medical personnel use information about the physical context of the user in order to make deductions regarding the user's state of health.

The physical state of the user can be picked up by sensors that convert the physical activity or the physiological signals into electrical signals that are processed by medical personnel. Tables I and II summarize the most common noninvasive sensors and the information that they provide for each user.

Over the past few years, several wearable health systems [14], [23] have been developed using a subgroup of the sensors included in Tables I and II, as determined by the particular objective of each system. Several projects based on wearable systems have been initiated to oversee the state of a person's physical health. The MyHeart [5] project focuses on the design of a personal health assistant (PHA) that is capable of the early and remote detection of atrial fibrillation, thus allowing, for example, immediate treatment involving medication. After a cerebral-vascular accident, neurological recovery can be stimulated by exercise and medication [24]. PHA wearable devices are necessary to capture the motor activities and evaluate the effects and efficacy of the treatment process. In [2], an ac-
TABLE II

SENSORS USED TO DETECT MOTION AND LOCATION

\begin{tabular}{ll}
\hline \hline \multicolumn{1}{c}{ Sensors } & \multicolumn{1}{c}{ Observation } \\
\hline Accelerometer & $\begin{array}{l}\text { Motion patterns of the body } \\
\text { and limbs }\end{array}$ \\
Microphone & $\begin{array}{l}\text { Speaker recognition, localiza- } \\
\text { tion by ambient sounds, activ- }\end{array}$ \\
& ity detection, speech features \\
Visible light sensor & Location of light sources \\
Rotation & Body movements \\
Compass & Orientation of the body and the \\
& head \\
Air Pressure & Vertical motion in elevator or \\
Light sensor & staircase \\
Environment temperature & Sunshine, location of lamps \\
Humidity & Outdoor, indoor \\
WLAN / GSM / CDMA & Location, weather conditions \\
Bluetooth, ZigBee & Location, user environment \\
\hline \hline
\end{tabular}

celerometer attached to an elastic belt placed over the pectoral region makes it possible to evaluate the recovery of motor skills and the efficiency of physical therapy for hemiplegic patients after an accident.

In [25], the mobile personal trainer (MOPET) system is proposed, a wearable system that supervises a physical fitness activity based on alternating jogging and fitness exercises in outdoor environments. By exploiting real-time data coming from sensors, knowledge elicited from a sport physiologist and a professional trainer, and a user model that is built and periodically updated through a guided autotest. Bamberg et al. [26], use wearable inertial sensors for motion classification and tracking in prior work, for example, in monitoring the activity of people at home, mainly for medical purposes. Leung et al. [27] present an information-processing paradigm for intelligent body sensor networks. Nodes in sensor networks have different levels of autonomy in terms of the signal processing, information fusion, and situation assessment in order to contribute with the overall system decision making. This approach is based on the use of a genetic algorithm to provide learning features to the sensor nodes, and fuzzy cognitive maps to perform situation assessment. Lee and Chung [28] propose a smart shirt to measure ECG and acceleration signals for continuous and real-time health monitoring. The measured physiological ECG data and physical activity data are transmitted in an ad hoc network in IEEE 802.15.4 communication standard to a base station and server PC for remote monitoring. Bickmore et al. [29] focus on the development of a handheld health counseling agent designed to promote physical activity is described. They present general-purpose software architecture for the rapid design and deployment of mobile health counseling agents. In [30], a general architecture for a wearable sensor system that can be customized to an individual patient's needs is presented. The architecture is based on embedded artificial intelligence that permits autonomous operation, sensor management, and inference, and may be applied to a general purpose wearable medical diagnostics. All the revised approaches discuss WSN and its 
application to wearable physiological monitoring and its applications [31]. However, they are very general and do not provide coordination and organization mechanisms, and are difficult to adapt to the changes that occur in the environment.

Current proposals have certain details in common, such as remote monitoring, although each one has a specific purpose. For example, some systems focus on dependent environments, while others focus on creating and integrating new sensors or applications that use wearable devices. All systems have the common objective of improving overall living conditions for patients and include remote monitoring systems for medical personnel. However, it is necessary to provide new solutions capable of facilitating the integration of smart wearable devices in intelligent environments. Current proposals focus on very specific problems and are a bit constrained when dealing with a dynamic environment. Intelligent environments require new integral solutions that can adapt to the changing conditions of the environment and adjust to the needs of the users.

MASs [32] has proven very suitable for modeling intelligent environments, given that they are based on social organizations and include learning capabilities. Furthermore, they can model smart wearable devices as agents within the system, thus making it easy to adapt to changes and to model the system agents as an active part of an organization. The MAS proposed in this research offers intelligent services to patients within their own surroundings, managing their problems, handicap, or illness in a noninvasive way. The MaRV system also keeps system users identified and located, so that the medical personnel incharge can remotely monitor the patient at any given time. To do so, the system relies on a ZigBee WSN that is used with lowconsumption digital radios based on the IEEE 802.15.4 Standard for wireless personal area networks (WPAN) [32], [33]. MaRV functions include installation, activation, deactivation and update management, and the elimination of services, as well as the authentification, control, and supervision of the residents.

\section{MARV MAS}

MASs are distributed systems based on the cooperation of multiple autonomous agents [9]. MaRV is a MAS that has evolved from the THOMAS architecture [32] to facilitate the integration of agents and smart wearable devices via wireless networks and mobile technology. THOMAS [32] is a MAS architecture that is useful for the new generation of virtual organizations in open environments. It consists of agents that are capable of autonomous decision making, incorporate learning mechanisms, and are able to respond to events by planning and replanning in execution time. THOMAS is an open architecture that can easily incorporate any type of agent. The MaRV MAS is a specialized feature of the THOMAS architecture for intelligent environments that can address the need to improve techniques for obtaining resident and patient data, as well as assign diagnoses in hospital centers and geriatric facilities, and monitor all types of patients. The MaRV MAS is a distributed agent platform that uses a ZigBee WSN to establish remote communication between patients and caregivers.

All smart wearable devices in MaRV are based on RFID technology. These devices, which identify and contain information for the residents, are hidden within their clothes and uniforms, so as to not inconvenience the residents in any way. RFID readers and mobile devices with NFC technology are used to read the RFID tags. NFC is a protocol based on a short-range wireless interface. Communication is established between two entities. The protocol establishes a wireless connection between the network applications and the electronic devices. NFC functions on a $13.56 \mathrm{MHz}$ band, thus obviating the need for a user license. NFC always has a device that both initiates and monitors the conversation. NFC fuses the usability of the RFID technology chip with the portability and market penetration of mobile telephony. It uses an electromagnetic field to communicate with a mobile telephone, personal digital assistants (PDA), or laptop using an intelligent RFID tag, or with other devices to perform micropayments, exchange of information, and access control. Additionally, the patients wear a series of sensors throughout the body that form a body area network (BAN) with which it is possible to obtain information about the patient's state of health [21]. These sensor devices are very small and comfortable, so that the patients do not reject them. At the same time, energy consumption should be optimal to avoid having to replace the battery so often. The MaRV system not only gathers and stores user information, but it is also a proactive computing system, since it anticipates user needs. The use of proactive computing [21] and smart wearable devices allows MaRV to provide the user with a degree of independence, and enriches the quality of life for both patients and caregivers.

As illustrated in Fig. 1, the MaRV system distinguishes three separate levels, each of which is in-charge of a specific functionality for the system.

1) The first level is comprised of the set of wearable sensors that capture patient information.

2) The second level is for data and information storage. It is made up of various types of devices (PDA, portable equipment, mobile telephones, etc.) that provide the computational capacity that allows data to be stored and processed prior to being used at the next level.

3) The third level is the medical WSN that registers and analyzes information to detect anomalies. This network acts as a medical services provider in cases, where the medical staff can handle emergency situations.

Different types of agents work on each of the system levels, as shown in Fig. 2. The MaRV MAS is based on a belief, desire, and intention (BDI) model [14], [15] in which the agent's function as controllers and coordinators for various medical care tasks. The agents can initiate services on demand, or according to planned actions or user requests. The behavior of each agent can change with any modification made to a patient's diagnosis. The same agents can modify patient treatment and respond to the smart wearable devices installed in the environment. Additionally, the information on the state of the system is continuously stored in order to allow for recovery in the event of system failure.

The different types of agents illustrated in Fig. 2 are as follows.

1) Catcher agents: These agents manage the portable RFID readers in order to obtain information from the RFID tags. There is one catcher agent for each type of information 

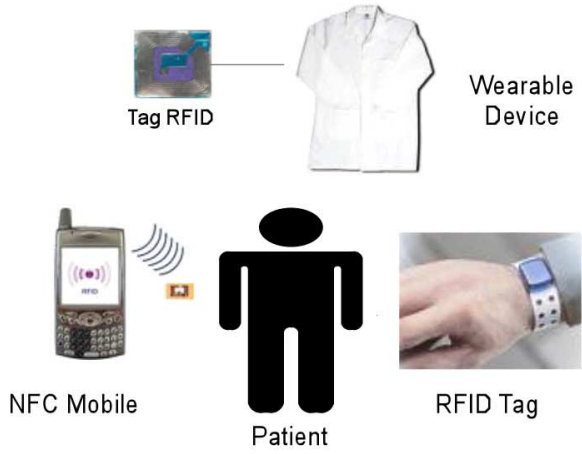
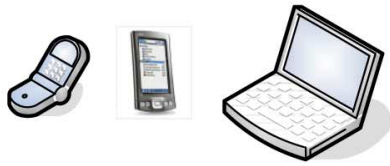

Portable Devices
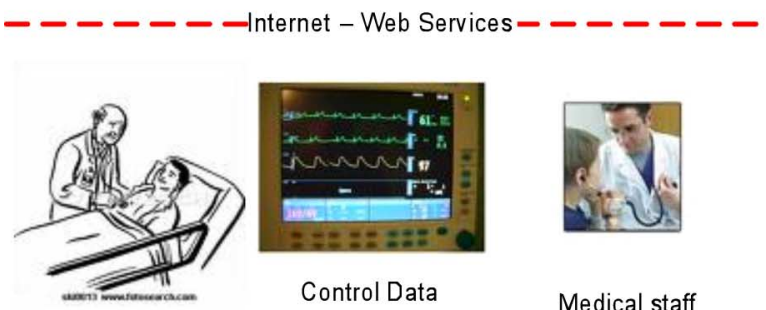

Caregivers

Fig. 1. Devices for each level of the MaRV system.

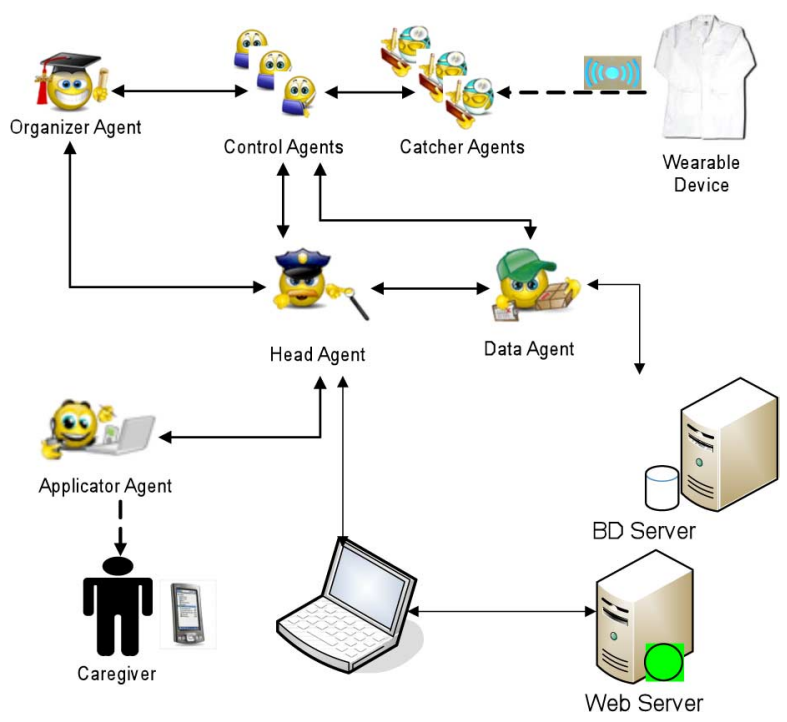

Fig. 2. MaRV system agents.

(location, identification, security, access control, etc.) that is captured in the system. There is also a catcher agent installed in mobile devices with NFC technology to gather information from the RFID tags, which it sends to other agents via Bluetooth, GPRS, or the ZigBee WSN.

2) Control agents: These agents supervise the activities of each of the catcher agents with the help of control direc- tives, and determine the validity of the information that is provided. They also classify the information received from the catcher agents, organize it and send it to the data agent and organizer agent. Moreover, they administer the plans generated by the organizer agent and ensure the plans are carried out by sending the information to the catcher agents. Finally, they inform the head agent about all of the incidents that arise during their processing.

3) Data agent: It structures and stores the information that it receives from the control agents in the information system. This effort facilitates the work of the organizer agent. The stored information can be consulted via the Internet.

4) Organizer agent: It automatically generates supervision plans and simple patient diagnoses. These plans are sent to the head agent, which is in-charge of distributing them between the control agent and the applicator agent.

5) Head agent: It controls the rest of the system agents. Manages the activation or deactivation of the control agents. Also receives the incident reports from the other agents (database errors, nonexecuted plans, security notifications, connections and disconnections by the control agents and catcher agents, etc.). The head agent sends this information (incident, date, time, system state, device affected, and detecting agent) to the data agent to be registered in the information system. Additionally, the head agent is responsible for informing the applicator agent about these incidents, according to their priority level.

6) Applicator agent: It administers the communication with the caregivers and medical personnel. Receives incident reports from the head agent, as well as information on the supervision tasks that have been carried out with the patients. All of this information can be sent via Bluetooth, Wi-Fi, ZigBee, or GPRS. In the event of any communication error, the head agent is informed, so that the information can be resent at a later time.

The organizer agent in MaRV integrates case-based reasoning (CBR) and case-based planning (CBP) mechanisms, which allows the agents to use previous experiences to create better plans and achieve their objectives. CBR [9] is a type of reasoning based on previous experiences. CBR systems solve new problems by adapting solutions that have been used to solve similar problems in the past, and learn from the new experiences. CBP is specifically designed to generate plans (action sequences) [14] from past experiences in execution time. In CBP, the proposal for solving a problem is a plan. This solution is generated after taking into consideration the plans that have been applied to solve similar problems in the past. The problems and their respective plans are stored in a memory of plans. The reasoning mechanism generates the plans that it wants to use from past experiences and planning strategies, which is how the concept of CBP is understood [15].

\section{A. Reasoning Agent Design}

Agents that are designed and implemented by CBR systems can reason autonomously and adapt to changes in their environment [15]. These abilities satisfy two of the most important 
characteristics for the organizer agent, as mentioned in the previous point: 1) reasoning ability and 2) facility in adapting to its surroundings. The organizer agent receives data from the sensors, as well as the patient profile and his medical treatments and adapts the tasks (plans) performed by the control agents, i.e., it adapts the organizational structure of the MAS. The other important characteristic is the ability to use the other agents to gather information from its environment. Therefore, in order to design and implement the organizer agent, it is necessary to consider the exchanges of information among the system agents. The IEEE Foundation for Intelligent Physical Agents (FIPA) ${ }^{1}$ specificationcan be considered as a valid standard for the communication between agents. To design the head agent, the Agent Unified Modeling Language (AUML ${ }^{2}$ ) methodology was used, since it provides the mechanisms required to obtain a design that is detailed enough to simplify the implementation phase.

The BDI model is a solid foundation for modeling and applying the internal behavior of the agents. The BDI model allows us to perceive the agent as an entity that is searching for an objective and behaves rationally. The CBR system and BDI agents can be connected if cases are implemented as beliefs, intentions, and desire that lead to the resolution of a problem. For a smooth transition between the design phase and the implementation phase, the CBR-BDI paradigm must be supported in the implementation phase.

The JavaAgent development framework (JADE) [34] is a good option for developing agent-based applications. JADE eXtension (Jadex) [35] is the implementation of a hybrid agent (reactive and deliberative) architecture for representing the state of JADE agents that follow the BDI model. Jadex is designed to be easily integrated into JADE by simply adding a packet. The primary objective is to facilitate the use of reasoning concepts during the implementation.

As seen from the outside, the organizer agent is a black box that receives and sends messages. The functioning of the organizer agent can be seen in greater detail by using the figure as following. The organizer agent, as previously described, is implemented as a Jadex agent. To do so, some variations in the Jadex architecture are introduced, as shown in Fig. 3.

Fig. 3 provides a summary of the architecture for the organizer agent. All messages received by the organizer agent, as well as internal events and new goals, are the first steps toward the internal reactions, and the deliberative and reasoning mechanisms processed by the organizer agent. The most significant new feature of the organizer agent's design, as seen in Fig. 3, is the integration of a CBR reasoning engine and a reactive system that gathers data from the sensors and control systems. This makes the design unique in its conception and reasoning capabilities. Based on the results from the CBR reasoning engine, the organizer agent sends plans through the CBP MaRV Plans. These plans can be executed immediately as events, or new plans can be generated and stored in the MaRV library to be executed at a future time. The execution of plans can modify the base of data sensors beliefs, send messages to other agents,

\footnotetext{
${ }^{1}$ www.fipa.org.

${ }^{2}$ www.auml.org.
}

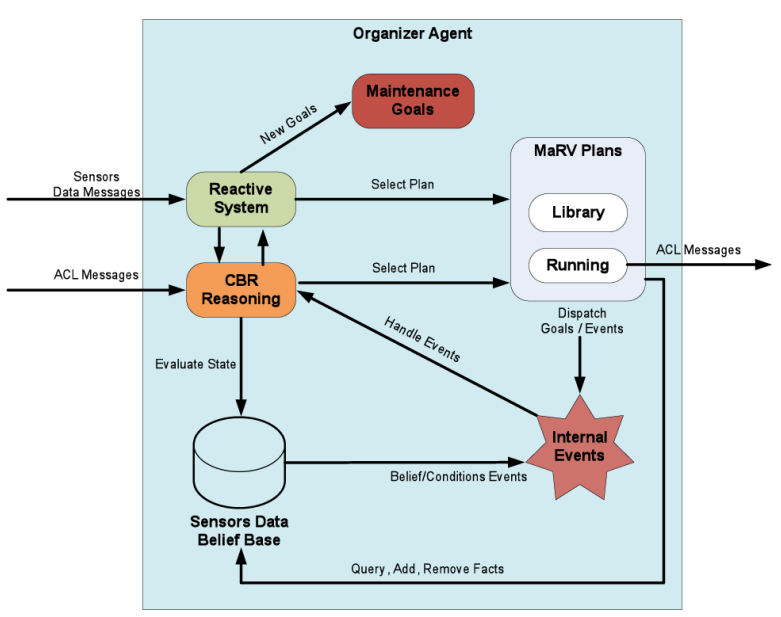

Fig. 3. Overview of the organizer agent architecture.

create new maintenance goals, or produce internal events. These action plans can respond to sensors installed in the system and facilitate the user's daily tasks, making the time spent by the user in the environment much more comfortable. The plans are used by different types of MaRV system agents that manage the active devices. The functionality implemented in Java classes can also be incorporated in other similar systems. The next section presents a low-level AUML design for the organizer agent, followed by the implementation of Jadex. One specialization of the CBR-BDI agent is the CBP-BDI agent [36]. Its internal structure is similar, but it differs from the CBR-BDI agent in which the solution that the agent carries out is not a model, but an actual plan, i.e., a sequence of actions. A CBP-BDI agent should incorporate a planning mechanism that generates plans as solutions to the given problem. A CBP-BDI agent can incorporate different planning mechanisms [36], [37]. Corchado et al. [36] present a replanning mechanism for CBP-BDI agents in which the main characteristic is the ability to replan in execution time. A CBP-BDI agent calculates the plan or intention that is most easy to replan: most replannable intention (MRPI). This is the plan that can most easily be replaced by another plan in case it is interrupted (for example, if a user changes preferences while the plan is being executed). In a dynamic environment, such as an agent society, the ability to have alternate plans is very important for maintaining efficiency within the system.

As shown in Fig. 4, the AUML design produces a diagram of classes for the organizer agent, the most important agent within the MaRV architecture. The agent has five capabilities and four services, as described in Fig. 4. The capabilities are: 1) P-solution; 2) C-sensor; 3) S-plans; 4) St-data; and 5) E-result. The services are: 1) provide information; 2) describe plan; 3) provide plan result; and 4) component task assignment.

Table III shows an example of the inputs received by the organizer agent. This information, together with the agent profile composes the problem description for the case. As can be seen in Table III, the organizer agent receives data from temperature, respiration, accelerometers, ECG sensors, and RFID tags that capture information from the patient. Every sensor provides a different type of data, for example, the temperature sensor provides a concrete measure, and the accelerometer provides 


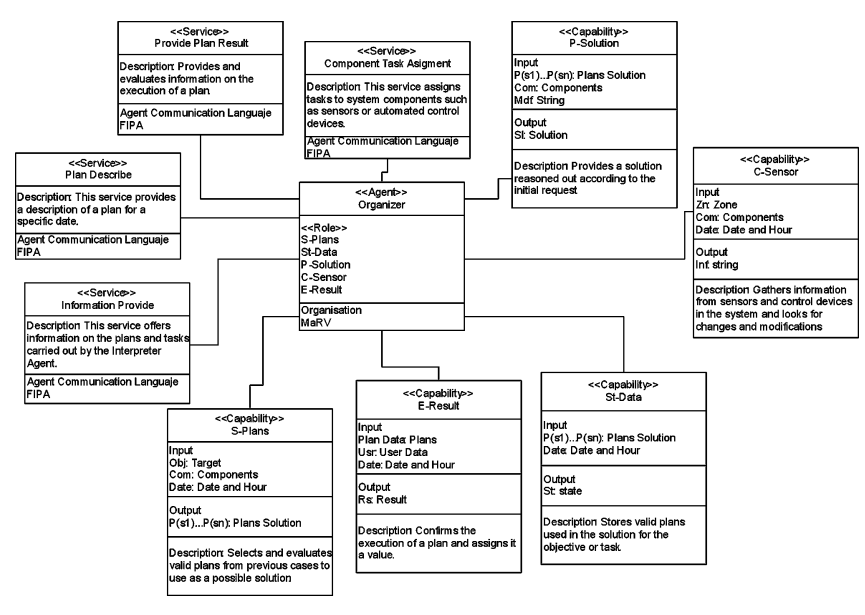

Fig. 4. Diagram of the different classes for the Organizer Agent.

TABLE III

EXAMPLE OF DATA RECEIVED BY THE ORGANIZER AGENT

\begin{tabular}{|c|c|c|c|c|}
\hline Sensors & Data & onOff & Date & Location \\
\hline $\begin{array}{l}\text { devicet01 } \\
\text { temperature }\end{array}$ & 36,5 & On & $\begin{array}{c}20100111 \\
20: 43: 21\end{array}$ & User01 \\
\hline $\begin{array}{l}\text { devicerf02 } \\
\text { RFID Tag }\end{array}$ & - & Off & - & User02 \\
\hline $\begin{array}{l}\text { devicea03 } \\
\text { accelerometer }\end{array}$ & 0 & On & $\begin{array}{c}20100111 \\
20: 43: 44 \\
\end{array}$ & User01 \\
\hline $\begin{array}{l}\text { deviceec04 } \\
\text { ECG }\end{array}$ & 0 & On & $\begin{array}{c}20100111 \\
20: 45: 29\end{array}$ & User01 \\
\hline $\begin{array}{l}\text { devicer05 } \\
\text { respiration }\end{array}$ & 85 & On & $\begin{array}{c}20100111 \\
20: 49: 01\end{array}$ & User01 \\
\hline
\end{tabular}

TABLE IV

SOLUTION GIVEN TO THE CASE

\begin{tabular}{lc}
\hline Description Field & Field Type \\
\hline Caseld & Integer \\
Patient Diagnosis & Diagnosis \\
Tasks & ArrayList of Tasks \\
Restrictions & ArrayList of Restriction \\
\hline
\end{tabular}

an alert when a fall is detected. The organizer agent manages these data in order to maintain the objectives for the patient. If the organizer agent detects any deviation or incident, it will be necessary to execute action plans or events.

The organizer agent analyzes the data received from the sensors, as well as the patient profile and his medical treatments. Taking into account these data, the organizer agent provides a patient diagnosis and evaluation and, according to the diagnosis, suggests a series of tasks (a plan) that should be performed, as shown in Table IV. Table IV describes the solution given to the case. As shown in Table IV, to assign the tasks to the control agents, it is necessary to take into consideration the possible restrictions of the patient's treatments.

\section{CASe Study: Geriatric Residences Monitoring}

This case study developed a multiagent MaRV system prototype in a geriatric care facility, which improves the quality of life for the patients, while simplifying and automating the work of the PHA in a geriatric care facility. This system maintains continual identification and location of the patients and PHA within the dependent environment. Additionally, it automatically carries out planning and task distribution among the PHA. The information that the system gathers from the sensors, and the actions carried out by the patients on a daily basis are all stored and processed by the system in order to be able to provide a diagnosis or alert of possible incidents at a later time.

The system uses mobile devices (PDA, mobile telephones, and NFC) and wireless communication technology (Wi-Fi, GPRS, RFID, NFC, and ZigBee) to interact with the users. These technologies and devices work in a distributed way, providing the users with a greater flexibility and easier access to the system services.

The standard ZigBee permits more than 65000 nodes to be connected in a network mesh topology. There are three types of ZigBee nodes: coordinator, router, and end device. The ZigBee coordinator controls the network and the connection method with the other devices. The ZigBee router interconnects devices, which are separated. The ZigBee end devices can communicate with the coordinators or the routers in order to transmit information, but they cannot exchange information among themselves. The ZigBee end devices remain in sleep mode in order to save energy and maximize their battery life.

The RFID technology [13] used in the development of this prototype allows us to automatically capture data that can be used to electronically identify patients, caregivers, and material resources. A RFID system is essentially comprised of two components: tags and readers. The tags can be passive (with no battery) or active (with battery). Active tags can be read by a reader at a greater distance than the passive tags. The RFID tag reader, which can be a mobile phone with NFC technology, gathers information as it moves throughout the tag's reading area, which it then transmits to the system. This information is comprised of the identification for the RFID tag that is being read, the identification of the reader that is being used, and the information contained in the RFID tag. The NFC mobiles transmit this information to the system via Bluetooth or GPRS, while the RFID readers use Wi-Fi to transmit the information via the internal network installed within the context.

Fig. 5 shows the installation of the ZigBee wearable sensor network in a geriatric care facility that can monitor both patients and PHAs. Each catcher agent captures information provide by a sensor and the control agent supervise the catcher agents. The data, organizer, head, and applicator agents are executed in a central computer. The ZigBee sensors that were installed identify and locate the patients and the PHA as they pass by strategically placed points. For example, the sensors located at the entrance door to the dining area identify the users that walk past and inform the system. This information is registered at both the entrance and exit area in order to control the schedule for each patient and possible missed meal that might weaken the patient's health. The installed RFID tags identify both material resources (beds, wheelchairs, sofas, tables, laptops, etc.) and human resources in the care home. For example, each caregiver is identified and located at every moment by means of a personal 


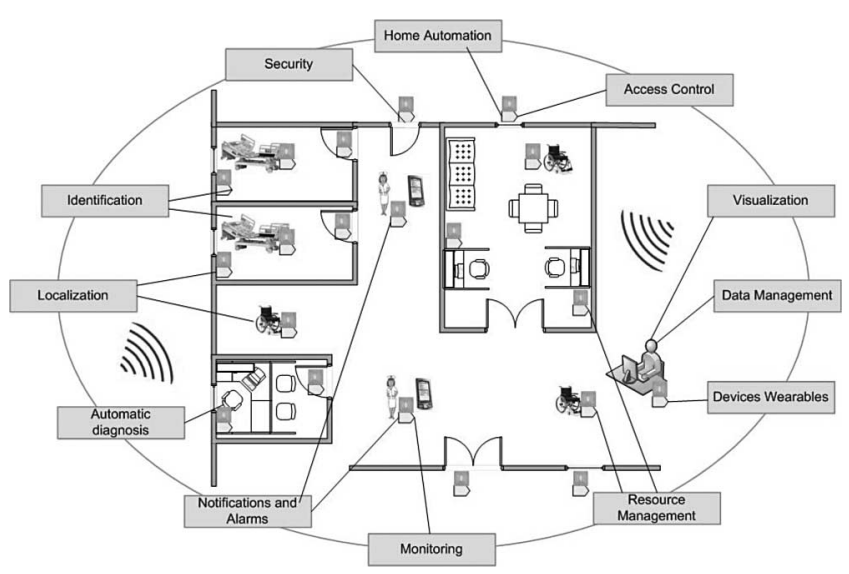

Fig. 5. ZigBee wearable sensor network in a geriatric care facility.

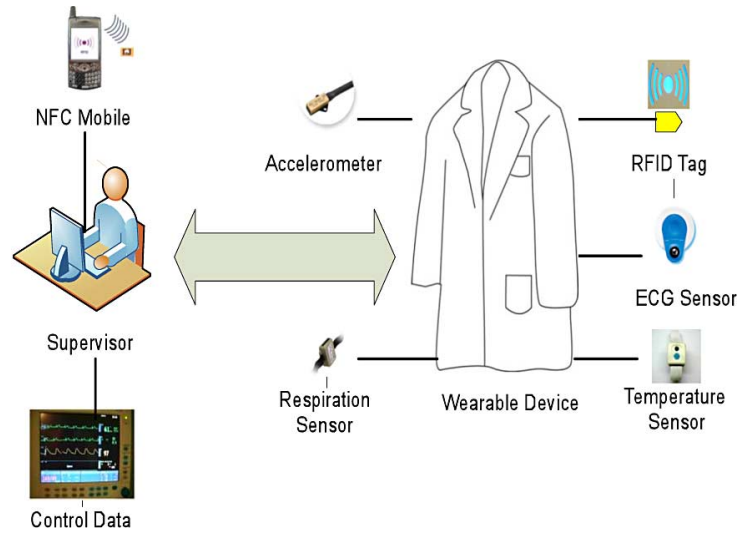

Fig. 6. Characteristics of the wearable device.

RFID tag. Each caregiver is also equipped with a PDA to which the system supervisor can send tasks dynamically via the internal network depending on the caregiver's particular situation and the task at hand. The caregiver can also input information on the state of the patients using a PDA. This information is automatically stored and process by the system, so that specialized supervisors or the system itself can perform remote diagnoses.

Fig. 6 illustrates the clothing used by the patients in the case study. It incorporates wearable devices, a RFID tag, an ECG sensory, a body temperature sensor, an accelerometer, and a respiration sensor. Each of these devices transmits the information gathered from the patient to the corresponding catcher agent via the ZigBee WSN. The RFID tag locates the patients and identifies them in the access control for the dining area, entertainment rooms, security areas, and bedrooms. The ECG sensor is applied with a strip of velcro to an area of the patient's clothing close enough to measure the heartbeat with an electric frequency. The MaRV system gathers the signal transmitted by the ECG sensor with a sampling frequency level of $200 \mathrm{~Hz}$. The signals received by the ECG can diagnose cardiovascular diseases or metabolic alterations. A triple axis accelerometer gathers signals on the patient's movements. The body temperature sensor monitors the patient's temperature. This sensor is an electronic device with a long-life battery. It gathers new patient data every $60 \mathrm{~s}$. The respiration sensor is another type of electronic device with a long-life battery that also gathers information every $60 \mathrm{~s}$. This
TABLE V

Comparison of Control Functions in the Home CARE Facility Before AND AFTER IMPLEMENTING THE MARV SYSTEM

\begin{tabular}{lcccc}
\hline \hline Sensors & $\begin{array}{c}\text { Control } \\
\text { escapes }\end{array}$ & $\begin{array}{c}\text { Control } \\
\text { Medication }\end{array}$ & $\begin{array}{c}\text { Control } \\
\text { Food } \\
\text { Intake }\end{array}$ & $\begin{array}{c}\text { Anomalies } \\
\text { in Patient } \\
\text { behavior }\end{array}$ \\
\hline $\begin{array}{l}\text { Before } \\
\text { MaRV }\end{array}$ & 63 & 92 & 88 & 46 \\
$\begin{array}{l}\text { After } \\
\text { MaRV }\end{array}$ & 98 & 100 & 100 & 95 \\
\hline \hline
\end{tabular}

particular frequency synchronizes perfectly with the definition of the respiratory frequency, such as the number of breaths per minute, and offers a satisfactory resolution of frequency and precision.

MaRV is a specialized feature of the THOMAS architecture [32] to facilitate the integration of agents and smart wearable devices via wireless networks and mobile technology. MaRV introduces compatibility with smart wearable devices through the use of ZigBee WSN and RFID and NFC technologies in order to establish remote communication between patients and caregivers. The primary characteristics of MaRV are the modeling capabilities for intelligent environments based on virtual organizations, the incorporation of special agents capable of interacting with smart wearable devices, the incorporation of agents with learning and adaptation capabilities, and the integration of a large variety of wireless technologies.

\section{RESUltS AND CONCLUSION}

Smart wearable technology [11] is particularly promising as a support for health care environments. Sensors and communication devices facilitate sending and receiving information in a ubiquitous and nonintrusive manner [38]. The MaRV system presented in this paper proposes an innovative technological solution consisting of an intelligent environment based on the use of smart wearable devices and MASs [15]. The MaRV system has been tested in a real environment, and the results obtained are promising. To validate the system, we designed a series of tests. The tests, which involved 50 patients, ten nurses, and two doctors, allowed us to evaluate the system. Specifically, the results of the MaRV system were studied over a period of five weeks.

The incorporation of new RFID and NFC sensors into the care home significantly improves a number of functions that include monitoring attempted escapes, controlling medication and food intake, and detecting anomalies in the patient's behavior, as shown in Table V.

Table $\mathrm{V}$ shows the percentage of success in controlling various functions within the care facility. It is easy to appreciate the efficiency of the MaRV system in controlling attempted escapes, allowing for an immediate response in $98 \%$ of the cases. Additionally, it is possible to control the medication and food intake of each patient with a $100 \%$ efficiency rate. The system makes it possible to avoid errors in the administration of medication and the special diets for the patients. Finally, the new sensor network makes it possible to detect anomalous behaviors among 


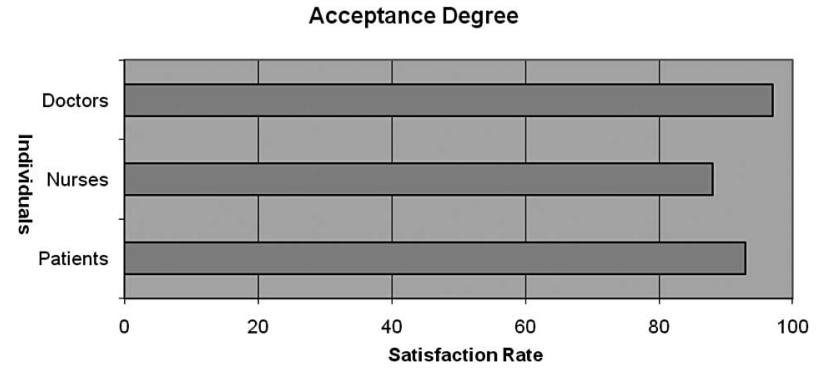

Fig. 7. Degree of acceptance of the MaRV system.

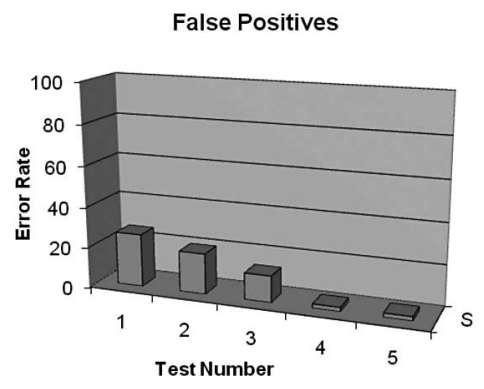

Fig. 8. False positives of the MaRV system.

demented patients and create behavior patterns for risky situations. The organizer agent takes into account past experiences in order to organize the new tasks in the system. In this sense, the MaRV system takes advantage of the previous experiences and learns these past lessons. This behavior provides a great capacity for adaptation, as demonstrate the results presented in Table V.

The degree of acceptance by the users involved in our system was very high, as shown in Fig. 7. The users completed surveys after using the system. Fig. 7 shows the results obtained after surveying the participants of the system. There was a 93\% acceptance rate among the patients, since the system is low invasive and does not affect their normal behavior. The acceptance among the nurses was $88 \%$, despite the initial learning phase. Nurses, who tried the system, demonstrated a certain rejection to the use of PDA, as well as to the training period required to learn the system. Finally, the acceptance by the doctors was $97 \%$. Users, in general, remarked the great capacity for dynamic adaptation that the system provides.

Fig. 8 shows the percentage of error that occurred in the escape control system during the tests. MaRV monitors the patients and detects possible escape attempts, which are managed by the head agent. The initial tests showed an error rate above $26 \%$. This percentage was primarily due to errors in the use of the system by the nurses. After the third test, the error rate was reduced to $13 \%$. From this point, the error rate remained stable at $2 \%$. This error could be reduced with a higher level of training in the use of the system. Furthermore, as previously indicated, the organizer agent uses a CBR strategy, and CBR systems typically require a period of time to learn from the environment, before providing accurate results.

Although the initial results are promising, the systems still require much work. The MaRV system has been successfully tested in a real environment and has proved to be an effi- cient monitoring mechanism that uses context-aware techniques based on MASs and smart wearable devices. Moreover, MaRV improved security to patients and caregivers by providing monitoring and alert facilities to detect and manage emergency situations. MaRV ensures that only authorized personnel have access to services and monitors the patients' treatments. Besides, the data is stored in a secure manner, using redundancy and continuous generation of backups.

However, the system presents some disadvantages on distributing the majority of the systems' functionalities into remote and local services, and applications. MASs present disadvantages when the number of agents is high. Current alternatives provide solutions, where the functionalities of the systems are not integrated into the structure of the agents. Our future work focuses on testing the system in different environments and looking for feedback to adjust and improve the proposed solution. Moreover, we will focus on distributing services and applications able to be invoked by the agents acting as controllers and coordinators. That is our next challenge.

\section{REFERENCES}

[1] G. D. Abowd, G. R. Hayes, G. Iachello, J. A. Kientz, S. N. Patel, M. M. Stevens, and K. N. Truong, "Prototypes and paratypes: Designing mobile and ubiquitous computing applications," IEEE Pervasive Comput., vol. 4, no. 4, pp. 67-73, Oct.-Dec. 2005.

[2] M. Akay, T. Tamura, Y. Higashi, and T. Fujimoto, "Unconstrained monitoring of body motion during walking," IEEE Eng. Med. Biol. Mag., vol. 22, no. 3, pp. 104-109, May/Jun. 2003.

[3] A. A. Freitas, D. C. Wieser, and R. Apweiler, "On the importance of comprehensible classification models for protein function prediction," IEEE/ACM Trans. Comput. Biol. Bioinformatics, vol. 7, no. 1, pp. 172182, Jan.-Mar. 2010.

[4] S. Wernicke, "Effcient detection of network motifs," IEEE/ACM Trans. Comput. Biol. Bioinformatics, vol. 3, no. 4, pp. 347-359, Oct.-Dec. 2006.

[5] MyHeart: Fighting cardio-vascular diseases by preventive lifestyle and early diagnosis. (2006) [Online]. Available: http://www.hitechprojects.com/euprojects/myheart/.

[6] J. Paradiso, G. Borriello, and P. Bonato, "Implantable electronics," IEEE Pervasive Comput., vol. 7, no. 1, pp. 12-13, Jan.-Mar. 2008.

[7] S. Giroux and H. Pigot, From Smart Homes to Smart Care: ICOST 2005 (Assistive Technology and Research). Fairfax, VA: IOS Press, 2005.

[8] B. Rhodes and K. Mase, "Wearable in 2005," IEEE Pervasive Comput., vol. 5, no. 1, pp. 92-95, Jan.-Mar. 2006.

[9] P. Baronti, P. Pillai, V. W. C. Chook, S. Chessa, A. Gotta, and Y. F. Hu, "Wireless sensor networks: A survey on the state of the art and the 802.15.4 and ZigBee standards," Comput. Commun., vol. 30, no. 7, pp. 1655-1695, 2007.

[10] ESTO: Science and Technology Roadmapping: Ambient Intelligence in EverydayLife (AmI@Life), Joint Res. Centre/Institute Perspect. Technol. Stud., Seville, Spain, 2003.

[11] G.-Z. Yang, Ed., Body Sensor Networks. London, U.K.: SpringerVerlag, 2006.

[12] K. Hung and Y. T. Zhang, "Implementation of a WAP-based telemedicine system for patient monitoring," IEEE Trans. Inf. Technol. Biomed., vol. 7, no. 2, pp. 101-107, Jun. 2003.

[13] C. C. Lin, P. Y. Lin, P. K. Lu, G. Y. Hsieh, W. L. Lee, and R. G. Lee, "A healthcare integration system for disease assessment and safety monitoring of dementia patients," IEEE Trans. Inf. Technol. Biomed., vol. 12, no. 5, pp. 579-586, Sep. 2008.

[14] J. M. Corchado, J. Bajo, Y. de Paz, and D. Tapia, "Intelligent environment for monitoring alzheimer patients, agent technology for health care," $D e$ cision Support Syst., vol. 34, no. 2, pp. 382-396, 2008.

[15] J. M. Corchado, J. Bajo, and A. Abraham, "GERAmI: Improving the delivery of health care," IEEE Intell. Syst., vol. 23, no. 2, pp. 19-25, Mar./Apr. 2008.

[16] B. Schön, G. M. P. O’Hare, B. R. Duffy, A. N. Martin, and J. F. Bradley, "Agent assistance for 3D world navigation," in Lecture Notes in Computer Science. vol. 1, New York: Springer-Verlag, 2005, p. 499. 
[17] M. O. Shafiq, Y. Ding, and D. Fensel, "Bridging multi agent systems and web services: Towards interoperability between software agents and semantic web services," in Proc. 10th IEEE Int. Enterprise Distrib. Object Comput. Conf., IEEE Computer Society, Washington, DC, 2010, pp. 8596.

[18] G. T. Jayaputera, A. B. Zaslavsky, and S. W. Loke, "Enabling run-time composition and support for heterogeneous pervasive multi-agent systems," J. Syst. Softw., vol. 80, no. 12, pp. 2039-2062, 2007.

[19] E. Strömmer, J. Kaartinen, J. Pärkkä, A. Ylisaukkooja, and I. Korhonen, "Application of near field communication for health monitoring in daily life," in Proc. 28th IEEE EMBS Int. Conf., New York City, NY, 2006, pp. 3246-3249.

[20] R. Want, "An introduction to RFID technology," IEEE Pervasive Comput., vol. 5, no. 1, pp. 25-33, Jan.-Mar. 2006

[21] Y. Hsu, C. Yang, T. Tsai, C. Cheng, and C. Wu, "Development of a decentralized home telehealth monitoring system," Telemed. e-Health, vol. 13, no. 1, pp. 69-78, 2007.

[22] A. Lymberis, "Smart wearables for remote health monitoring, from prevention to rehabilitation: Current R\&D, future challenges," in Proc. 4th Annu. IEEE Conf. Inf. Technol. Appl. Biomed., 2002, pp. 272-275.

[23] A. Milenkovic, C. Otto, and E. Jovanov, "Wireless sensor networks for personal health monitoring," Issues and an Implementation, Comput. Commun., Wireless Sens. Netw. Wired/Wireless Internet Commun., vol. 29, no. 13-14, pp. 2521-2533, 2006.

[24] J. Stein, "Wearable sensor technology for functional assessment after stroke," IEEE Eng. Med. Biol. Mag., vol. 22, no. 3, pp. 26-27, May-Jun. 2003.

[25] F. Buttussi and L. Chittaro, "MOPET: A context-aware and user-adaptive wearable system for fitness training," Artif. Intell. Med., vol. 42, no. 2, pp. $153-163,2008$.

[26] S. J. M. Bamberg, A. Y. Benbasat, D. M. Scarborough, D. E. Krebs, and J. A. Paradiso, "Gait analysis using a shoe-integrated wireless sensor system," IEEE Trans. Inf. Technol. Biomed., vol. 12, no. 4, pp. 413-423, Jul. 2008.

[27] H. Leung, S. Chandana, and S. Wei, "Distributed sensing based on intelligent sensor networks," IEEE Circuits Syst. Mag., vol. 8, no. 2, pp. 38-52, May 2008.

[28] Y. D. Lee and W. Y. Chung, "Wireless sensor network based wearable smart shirt for ubiquitous health and activity monitoring," Sens. Actuators B: Chem., vol. 140, no. 2, pp. 390-395, 2009.

[29] T. W. Bickmore, D. Mauer, and T. Brown, "Context awareness in a handheld exercise agent," Pervasive Moblie Comput., vol. 5, no. 3, pp. 226235, 2009.

[30] W. H. Wu, A. T. Bui, M. A. Batalin, L. K. Au, J. D. Binney, and W. J. Kaiser, "MEDIC: Medical embedded device for individualized care," Artif. Intell. Med., vol. 42, no. 2, pp. 137-152, 2008.

[31] P. S. Pandian, K. P. Safeer, P. Gupta, D. T. Shakunthala, B. S. Sundersheshu, and V. C. Padaki, "Wireless sensor network for wearable physiological monitoring," J. Netw., vol. 3, no. 5, pp. 21-29, 2008.

[32] J. Bajo and J. M. Corchado, "Thomas: Practical applications of agents and multiagent systems," in Proc. 10th Int. WorkConf. Artif. Neural Netw., Salamanca, Spain, LNCS, Berlin, Germany, 2009, pp. 512-513.

[33] Y. Huang and A. Pang, "A comprehensive study of low-power operation in ieee 802.15.4," in Proc. 10th ACM Symp. Model., Anal., Simul. Wireless Mobile Syst. (MSWiM 2007), Chania, Crete Island, Greece, New York, NY, 2007, pp. 405-408.

[34] Z. Z. Belloum, A. de Laat, C. Adriaans, P. Hertzberger, and B., "Using Jade agent framework to prototype an e-science workflow bus," in Proc. Cluster Comput. Grid. Seventh IEEE Int. Symp., Rio de Janeiro, 2007, pp. $655-660$.

[35] A. Pokahr, L. Braubach, and W. Lamersdorf, "Jadex: A BDI Reasoning Engine," in Chapter Multi-Agent Programming. Norwell, MA: Kluwer, 2005

[36] J. M. Corchado, M. Glez-Bedia, Y. de Paz, J. Bajo, and J. F. de Paz, "Concept, formulation and mechanism for agent replanification: MRP architecture," Comput. Intell., vol. 24, no. 2, pp. 77-107, 2008.

[37] J. Bajo, V. Julián, J. M. Corchado, C. Carrascosa, Y. de Paz, V. Botti, and J. F. de Paz, "An execution time planner for the ARTIS agent architecture," Eng. Appl. Artif. Intell., vol. 21, no. 5, pp. 769-784, 2008.

[38] Y. S. Yan and Y. T. Zhang, "An efficient motion-resistant method for wearable pulse oximeter," IEEE Trans. Inf. Technol. Biomed., vol. 12, no. 3, pp. 399-405, May 2008.

[39] G. L. Bodic, Mobile Messaging, Technologies and Services, 2nd ed. New York: Wiley, 2005.

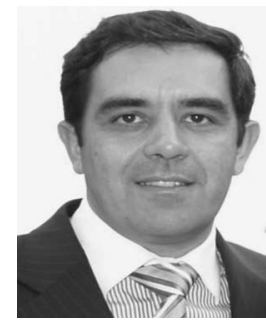

Juan A. Fraile received the Bachelors degree in information technology and master's degree in computer sciences from the Pontifical University of Salamanca, Salamanca, Spain, in 1996 and 2000, respectively, where he is currently working toward the Ph.D. degree.

He has been involved in the development of automated systems in the DMR Consulting Company and the Data Processing Centre, Pontifical University of Salamanca. He has also been a coauthor of papers published in recognized workshops and symposiums.

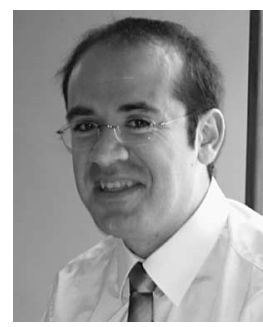

Javier Bajo received the Ph.D. degree in computer science from the University of Salamanca, Salamanca, Spain.

$\mathrm{He}$ is currently an Associate Professor at the Pontifical University of Salamanca, Salamanca, Spain. His research interests include multiagent systems, decision support systems, and intelligent environments.

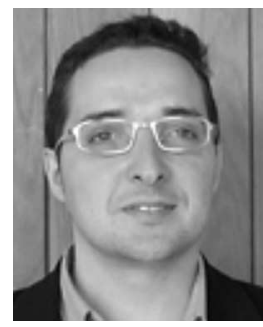

Juan M. Corchado received the Ph.D. degree in computer science from the University of Salamanca, Salamanca, Spain, and the Ph.D. degree in artificial intelligence from the University of Paisley, Paisley, U.K.

$\mathrm{He}$ is currently the Dean of the Faculty of Computer Science and an Associate Professor at the University of Salamanca, where he is also engaged with the Biomedicine, Intelligent Syatems and Educational Technonlogy Research Group and the M.Sc. programs in computer science. Since 1993, he has also been a Research Collaborator with the Plymouth Marine Laboratory, Plymouth, U.K. His research interests include agents and multiagent systems, ambient intelligence, case-based reasoning, and real-time forecasting.

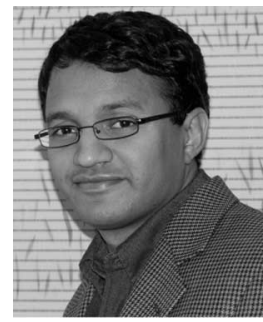

Ajith Abraham (M'96-SM'07) received the M.S degree from Nanyang Technological University, Nanyang, Singapore, and the Ph.D. degree in computer science from Monash University, Melbourne, Australia.

He is currently a Coordinator with the Machine Intelligence Research Labs, Scientific Network for Innovation and Research Excellence, Washington, DC, which has members from more than 60 countries. He has a worldwide academic experience with formal appointments in Monash University; Oklahoma State University, Stillwater, OK; Chung-Ang University, Seoul, Korea; Jinan University, Jinan, China; Rovira i Virgili University, Tarragona, Spain; Dalian Maritime University, Dalian, China; Yonsei University, Seoul, Korea; the Open University of Catalonia, Barcelona, Spain; the National Institute of Applied Sciences, Lyon, France; and the Norwegian University of Science and Technology, Trondheim, Norway. He has been engaged with the editorial board of several reputed international journals. He has also been the Guest Editor of 35 special issues on various topics. He has authored or coauthored more than 600 publications. His research and development experience includes more than 20 years in the industry and academia. He has given more than 35 plenary lectures and conference tutorials in various fields. His current research interests include machine intelligence, terrorism informatics, network security, sensor networks, e-commerce, web intelligence, web services, computational grids, data mining, and their applications to various real-world problems.

Dr. Abraham is a Senior Member of the IEEE Systems Man and Cybernetics Society, the IEEE Computer Society, the Institution of Engineering and Technology, U.K., the Institution of Engineers Australia, Australia, etc. He is a Co-Chair of the IEEE Systems Man and Cybernetics Society Technical Committee on Soft Computing. He is actively involved in the Hybrid Intelligen Systems, Intelligent Systems Design and Applications, Information Assurance and Security, and Next Generation Web Services. He is also the receipient of best paper awards at international conferences. He was also with the Practices (NWeSP) series of international conferences, in addition to, other conferences. 